\title{
Apprendre les langues au XVI siècle : Le Vocabulaire de trois langues, cestassavoir latine, italienne et francoyse
}

Nadia Minerva

\section{OpenEdition}

Journals

\section{Édition électronique}

URL : https://journals.openedition.org/dhfles/154

DOl : $10.4000 /$ dhfles. 154

ISSN : 2221-4038

\section{Éditeur}

Société Internationale pour l'Histoire du Français Langue Étrangère ou Seconde

\section{Édition imprimée}

Date de publication : 1 juin 2009

Pagination : 11-27

ISSN : 0992-7654

Référence électronique

Nadia Minerva, «Apprendre les langues au XVIe siècle : Le Vocabulaire de trois langues, cestassavoir latine, italienne et francoyse », Documents pour l'histoire du français langue étrangère ou seconde [En ligne], 42 | 2009, mis en ligne le 16 janvier 2011, consulté le 14 juin 2021. URL : http:// journals.openedition.org/dhfles/154; DOI : https://doi.org/10.4000/dhfles.154

Ce document a été généré automatiquement le 14 juin 2021.

(c) SIHFLES 


\title{
Apprendre les langues au XVI siècle : Le Vocabulaire de trois langues, cestassavoir latine, italienne et francoyse
}

\author{
Nadia Minerva
}

1 La présente étude a pour but d'ajouter une petite tesselle à la mosaïque du plurilinguisme du $\mathrm{XVI}^{\mathrm{e}}$ siècle : évoquant le sujet bien connu de la valorisation des langues et des cultures nationales au début de l'époque moderne, elle se focalisera sur les rapports entre elles dans les dictionnaires et les vocabulaires polyglottes notamment sur la question de la hiérarchie qui y est établie - et sur la circulation de ces outils, outils qui se recoupent souvent mais qui présentent tout aussi souvent des traits originaux.

2 Le Vocabulaire de trois langues ${ }^{1}$, présenté dans ces pages, se prête à une réflexion sur les facteurs d'expansion des langues nationales et sur l'intense activité plurilingue qui en constitue le corollaire ; il peut donc aider à mieux tracer les axes portants de la polyglossie à un moment de notre histoire culturelle où les parlers vernaculaires sont utilisés de plus en plus pour les contacts entre des communautés linguistiques voisines ; ce qui fait naître l'exigence de les apprendre.

3 Tous les outils qui ont permis d'apprendre les langues au $\mathrm{XVI}^{\mathrm{e}}$ siècle ne sont pas connus. Il y en a sans doute plusieurs qui ne nous sont pas parvenus ou qui restent ensevelis dans les bibliothèques privées. Quand d'heureux hasards permettent d'en rencontrer un et qu'on l'examine, on se rend compte que même l'outil le plus modeste a beaucoup à nous livrer sur l'enseignement/apprentissage des langues et sur l'idée de plurilinguisme d'autrefois, et qu'il peut aussi nous renseigner sur les hypersensibilités (voire, les idiosyncrasies) linguistiques d'aujourd'hui.

4 En effet, ce répertoire lexicographique trilingue fournit l'occasion de se poser quelques questions non marginales :

1. par quels véhicules linguistiques les langues modernes se sont-elles répandues? 
2. quels rapports se sont instaurés entre deux vernaculaires romans, l'italien et le français, rapports entre eux et rapports de ces langues romanes avec le latin? Question d'où en découle une autre :

3. quel est le rôle du latin dans ces contacts entre les langues vulgaires?

4. à quel moment, sous l'impulsion de quels facteurs et par quels moyens le plurilinguisme de la Renaissance a-t-il commencé à décliner laissant la place aux différents bilinguismes et monolinguismes ? Et, enfin, dernière question qui anticipe déjà mon hypothèse :

5. quel rôle a joué la normation des langues modernes dans ce déclin?

5 Le point de départ de mon parcours est justement ce dictionnaire trilingue qui s'est révélé un outil chargé de mystères et dont les anomalies par rapport aux autres outils de l'époque sont frappantes.

\section{Les énigmes}

Notre vocabulaire - repéré à la Réserve de la Bibliothèque Nationale de France - est inconnu ou presque : il ne figure ni dans les volumes consacrés aux éditions du XVI siècle, éditions pourtant bien étudiées, ni dans la «bible des chercheurs ", à savoir les répertoires que Philippe Renouard a consacrés aux imprimeurs parisiens. À ma connaissance, il n'est mentionné que trois fois :

7 (1) dans le Catalogue Pichon (1897-1898, $1^{\text {ère }}$ partie : 205-206) où il figure sous le $n^{\circ} 709$. C'est cet exemplaire du baron Pichon, acheté par la BN en 1900, que j'ai consulté. Nicole Bingen dans son Maitre italien (1987) en reproduit la notice.

8 (2) Nicole Bingen s'aperçoit qu'il s'agit probablement du premier dictionnaire paru en France où l'on trouve conjointement le français et l'italien ${ }^{2}$. Elle avance une hypothèse convaincante sur sa datation et sur ses possibles imprimeurs : "Très petit octavo gothique $^{3}$,ce vocabulaire a dû probablement paraître avant la $1^{\text {ère }}$ édition parisienne en huit langues du Vocabulista (1546), chez la veuve de Jean Saint-Denis ou chez Pierre Sergent» (Bingen 1987 : 247). Dans une autre publication, cette hypothèse se précise : «publié probablement entre 1531 et 1545 » (Bingen / Van Passen 1991 : 3008). C'est le colophon qui a sans doute inspiré cette datation : «Cette adresse [rue Neuve NotreDame, à l'enseigne S. Nicolas] est celle de Jean Saint-Denis (1521-31), de sa veuve Claude (1531-1549), de Pierre Sergent avant de passer aux Bonfons » (Bingen : 1987 : 247).

9 (3) Enfin, Margarete Lindemann (1994) mentionne le Vocabulaire dans son répertoire des dictionnaires français parus avant 1600. Elle le date entre 1530 et 1550 (datation précise-t-elle (p. 659) - suggérée par les conservateurs de la Bibliothèque nationale de France). La présence de quelques formes verbales conjuguées lui suggère de le relier à la tradition du Vocabulista (la seule nomenclature plurilingue de l'époque): l'auteur anonyme aurait transformé les dialogues et les phrases de sa source en une liste de mots. Elle relève d'ailleurs quelques correspondances dans le lexique présenté.

En effet, si on ne connaît ni la date de parution ni l'éditeur du Vocabulaire, on peut du moins faire des conjectures : de nombreux imprimeurs et libraires parisiens de la rue Neuve Notre-Dame se sont servis de l'enseigne Saint Nicolas : Jehan Hérouf (1501-1528), Jehan Saint-Denys (1521-1531) et sa veuve Claude, Pierre Sergent (1530-1547) Jehan Bonfons (1547-1566), sa veuve et son fils Nicolas Bonfons (1572-1623). Mais dans le colophon de Jean Hérouf on lit : «à l'image Saint Nicolas » et non "à l'enseigne ». Quant aux Bonfons, il n'y a aucune trace de notre Vocabulaire dans leurs éditions qui ont 
été scrupuleusement recensées dans le mémoire d'Arlette Destot (1977); ce qui restreint considérablement la période plausible de publication du Vocabulaire. J'aurais donc tendance à accueillir l'hypothèse de Bingen / Van Passen, mais je n'exclus pas d'autres possibilités : imprimeurs dont on n'a pas conservé de trace, surtout si peu importants, non consacrés (pas de marque typographique dans le Vocabulaire, tandis que les éditions de Saint-Denys et de Pierre Sergent en comportent toujours), édition piratée, échanges de matériaux entre les divers imprimeurs, ce qui arrivait très souvent. Je me suis même demandé si on peut être sûr du lieu d'édition : si l'ouvrage avait été imprimé à l'étranger, la mention de Paris dans la page de titre aurait fourni de belles garanties de correction linguistique pour le français...

11 Les caractères gothiques - mieux la bâtarde réservée aux textes en langue vulgaire - ne fournissent pas non plus d'indices : ce sont bien des caractères qui ont tendance à disparaître, mais si le romain commence à s'imposer, bien des imprimeurs continueront à les utiliser jusque dans la seconde moitié du siècle.

12 Le Vocabulaire a le même format que le Vocabulista, comme toute la littérature populaire de l'époque : un livre de petites dimensions, de lecture facile, plus facile à lire, à manier, à colporter, et pas tellement destiné à figurer sur les rayons des bibliothèques - d'où la rareté des exemplaires.

13 On ne parviendra peut-être jamais à dévoiler les mystères qui entourent notre petit vocabulaire. Plusieurs problèmes restent ouverts et les questions sont plus nombreuses que les réponses. Pour ce qui concerne la datation du Vocabulaire, les hypothèses formulées par Nicole Bingen et Anne-Marie Van Passen, bien que basées sur une description externe relevant de l'histoire de l'édition plutôt que de l'analyse de son contenu linguistique et culturel, peuvent constituer un bon point de départ pour situer le Vocabulaire dans les années 1530-1550. Quant aux sources possibles de son compilateur, ses caractéristiques tout à fait singulières peuvent fournir quelques indices.

\section{Les anomalies}

14 Les particularités du Vocabulaire par rapport à la production plurilingue de l'époque sont frappantes. Pour mieux les saisir, il faut s'éloigner du Vocabulaire et tracer rapidement le contexte où il se situe.

15 L'activité lexicographique plurilingue qui caractérise le XVI ${ }^{e}$ siècle est marquée par la dépendance réciproque des différents recueils dans l'Europe toute entière. L'étendue de cette lexicographie témoigne de l'intérêt pour les langues modernes à plusieurs niveaux. Je me limite à évoquer les principaux secteurs de cette activité :

Un mouvement érudit qui se manifeste dans la description et la comparaison des langues ; que l'on pense, par exemple, pour ne citer que deux ouvrages célèbres, aux Institutiones linguae syriacae, assyriacae atque thalmudicae, una cum aethiopicae atque arabicae collatione d'Angelo Canini (Paris, 1554) et au Mithridate ${ }^{4}$ (Zurich, 1555) de Conrad Gessner.

17 La lexicographie savante. On n'en retiendra que deux titres célèbres : le Dictionarium d'Ambrogio Calepino (type du dictionnaire plurilingue alphabétique à entrée latine) et le Nomenclator d'Hadrianus Junius (type de la nomenclature plurilingue à entrée latine). 

Jugement d'amour ${ }^{5}$ de Juan de Flores (Grisel y Mirabella, fin $\mathrm{XV}^{\mathrm{e}}$ siècle), traduit de l'espagnol, d'abord en italien, puis en français (à partir du texte italien), qui connaît au moins 12 autres éditions françaises entre 1530 et 1555, et 15 éditions bilingues italien / français entre 1546 et $1582^{6}$.

culturel. Dans ce domaine on recense deux types d'outils : le recueil de dialogues comportant un dictionnaire comme le Berlaimont (postérieur, en version polyglotte, à notre Vocabulaire) et les dictionnaires d'usage populaire connus sous le nom de Vocabulista.

C'est sur ces derniers que je porterai mon attention, car, à cette époque-là, les Vocabulista sont les seuls dictionnaires où l'on peut trouver, parmi d'autres langues, l'italien et le français. Ce sont donc les seuls outils qui permettent une comparaison avec le Vocabulaire de trois langues et c'est cette comparaison qui montre les anomalies de ce dernier.

21 La littérature critique nous dit que le premier dictionnaire plurilingue où figurent conjointement le français et l'italien est l'Introductio ${ }^{7}$, parue à Rome chez Mazzocchi en 1510. On compte des dizaines d'éditions de l'Introductio (que nous appellerons désormais Utilissimo Vocabulista, titre qui deviendra bientôt le plus répandu), un nombre croissant de langues (jusqu'à huit) et une étendue géographique surprenante ${ }^{8}$. En Italie, il est réédité - à Rome et à Venise - jusqu'en 1582 (12 éditions) ${ }^{9}$. Les éditions allemandes foisonnent. En France, avant l'édition novatrice de Le Tellier (Dictionnaire des huict langaiges, Paris 1546) ${ }^{10}$, on connaît deux éditions lyonnaises de 1533 et de 1542, en 5 langues, reliés à la filière italienne inaugurée par Francesco Garrone (voir note 9).

Le Vocabulista est une petite nomenclature organisée par champs sémantiques qui répond à une demande du marché, un outil pensé pour la communication internationale, indispensable, comme l'évoquent les différentes pages de titre, à ceux «qui vont praticant dans le monde » ("per mundum versari»), à ceux qui veulent apprendre les langues "sans aller ale scole [sic], come artisans, \& femmes», aux «persone di scarsa dottrina costrette a viaggiare in diverse nazioni », à «mercanti e passaggieri » pour se passer de l'interprète, «\& estraneo, \& forse inimico » : ce voyageur ne sera « costretto piu d'altri con suo perielio fidarsi »... Plus tard, d'autres destinataires viennent s'ajouter : les «studieux» et les «amateurs des lettres», dans les compilations en huit langues (en commençant par le grec), sans pour autant que n'y apparaisse aucune prétention savante : lexiques et phraséologies à la fois, ces outils répondent aux besoins de la communication courante. Jamais la norme, seulement l'exemple, et l'accent est toujours mis sur l'utile.

23 Comme le Vocabulista, le Vocabulaire est pratique par sa forme (colonnes, petit format) et par son contenu (lexique en majorité du quotidien). Cependant, les différences sont marquantes.

- Le Vocabulaire est disposé sur trois colonnes : on a souvent remarqué la rareté des dictionnaires trilingues au XVI ${ }^{\mathrm{e}}$ siècle, un nombre impair de langues posant des problèmes de mise en page. La mise en colonne est un métier spécialisé. Il faudrait trouver un imprimeur parisien ayant cette compétence, mais je n'en connais aucun, alors qu'il y en a dans l'imprimerie hollandaise ou allemande...

- Il est trilingue : si les trilingues sont rarissimes, on n'en connaît aucun qui enregistre l'italien et le français. 
- Il est alphabétique : le corpus que l'on connaît est constitué en grande partie de listes lexicales thématiques et de modèles conversationnels ; la nomenclature du Vocabulista est organisée par thèmes.

- Le public aussi est nouveau : aucune mention n'est faite aux marchands et aux voyageurs et la disposition alphabétique ne favorise pas ce type de consultant.

Le Vocabulaire de trois langues est donc une liste alphabétique de mots sur trois colonnes. Il se présente, en vertu de cette organisation macro-structurelle, comme un premier essai de contamination entre les deux traditions lexicographiques de l'époque : les dictionnaires populaires dont je viens de parler et les dictionnaires doctes, conçus pour les savants. L'ordre alphabétique est établi sur l'italien qui occupe la colonne centrale, entre le latin et le français. Nous sommes encore loin, bien sûr, des dictionnaires de la fin du siècle : l'ordre alphabétique ne concerne que la lettre initiale du mot ou du syntagme répertoriés. Rien de surprenant en cela : on enregistre encore des hésitations dans les grands classiques de la lexicographie du XVIe siècle. Voici ce que l'on trouve par exemple sous A :

\begin{tabular}{|l|l|l|}
\hline Latin & Italien & francoys \\
\hline Apostoli & Apostol & les apostres \\
\hline Nunc & Adesso & Maintenant \\
\hline Ambrosius & Ambroso & ambroise \\
\hline Ludouicus & Aloisi & Loys [...] \\
\hline Adam & Adamo & adam \\
\hline Adueniat & Auanga & aduiengne \\
\hline Autunnus & Autunno & automne \\
\hline Conuenire & Accordare & accorder [...] \\
\hline Aperi & Apre & Ouure \\
\hline Assetum & Assedo & Vinaigre [...] \\
\hline Ire & Andare & aller \\
\hline Lacerare & Astrazare & Dechirer [...] \\
\hline Anas & Anade & Cane \\
\hline Mihi & A my & A moy [...] \\
\hline Tangere & Atocare & Atoucher [...] \\
\hline
\end{tabular}


L'ordre alphabétique devient primordial pour déterminer les utilisateurs potentiels et leurs besoins linguistiques : dans les vocabulaires polyglottes de l'époque, c'est l'organisation onomasiologique qui guide l'utilisateur : il ne s'agit pas de trouver un mot, mais un groupe de mots relevant tous du même champ lexical, parfois liés à la même situation de communication. Le dictionnaire alphabétique s'adresse à un utilisateur qui veut trouver non pas un sujet mais un mot - dans notre cas un mot italien : le vocabulaire n'est pas réciproque. Dans notre proto-dictionnaire, il n'y a qu'une seule langue source (l'italien) et une seule langue cible (le français).

Qui peut avoir eu besoin de chercher un mot italien? Un Italien qui veut écrire ou parler en français? Un Français ou un étranger qui lit des textes italiens? Un traducteur? Difficilement le voyageur ou le marchand qui se repéraient mieux dans un recueil thématique. Et si notre vocabulaire était lui-même le résultat d'un travail de traduction? D'ailleurs, on le sait, les premières listes lexicales bilingues nous viennent des glossaires visant à faciliter la compréhension de textes devenus difficiles pour les lecteurs de leur époque.

Comme le précise le sous-titre, le Vocabulaire est destiné à « ceulx qui ont desir sçavoir de l'italien ou bien du françoys ", deux langues qui sont encore à portée communicative limitée sur la scène européenne, d'où la présence du latin qui devrait être la voie d'accès aux langues modernes, latin qui joue le rôle de lingua franca (rôle qu'il aura encore pour longtemps, inutile de le rappeler). Le recours à une langue à large diffusion, langue de prestige, langue de la communication internationale pourrait induire à penser qu'une hiérarchie s'établit dans le Vocabulaire; au contraire, le prestige du latin n'engendre pas de rapports asymétriques entre ces trois langues.

8 Pour ce qui concerne la nomenclature, le collationnement des deux seuls dictionnaires disponibles - l'Utilissimo Vocabulista et le Vocabulaire de trois langues - a montré leur parenté, bien que ce dernier enregistre plusieurs mots absents dans les Vocabulista et que le choix lexical opéré par le Vocabulaire lui donne un aspect assez nouveau ${ }^{11}$. Je me limite ici à des remarques concernant les rapports entre les langues en présence et ce plurilinguisme plus bigarré qu'on ne le croirait où ont égal droit de cité toutes les langues et tous les parlers.

\section{Variété linguistique et polyglossie}

Un des aspects intéressants du Vocabulaire de trois langues est la variété d'italien enregistrée. La question du plurilinguisme, de la codification et de la réflexion linguistique dans l'Italie des débuts du XVI ${ }^{e}$ siècle est trop complexe pour qu'on puisse la liquider sommairement. En Italie, on le sait, c'est la Babel des langues régionales, voire municipales. On n'a pas eu un Villers-Cotterêts, on ne peut pas parler de politique linguistique, mais un mouvement culturel qui gagne le consensus de la République des Lettres valorise de plus en plus le toscan des Trois Couronnes (Dante, Pétrarque, Boccace) au détriment de langues de prestige, même littéraires, comme par exemple les parlers du Nord de l'Italie ${ }^{12}$. Les débuts de l'activité lexicale en Italie donnent une poussée importante à l'assimilation pétrarquiste de la langue ; mais l'Italie des Cours a du mal à s'y résigner et les tenants de la «lingua cortigiana » sont légion. Notre Vocabulaire ne semble pas tenir compte de ces affrontements : 


\begin{tabular}{|l|l|l|l|l|}
\hline \multicolumn{4}{|l|}{ Vocabulaire de trois langues } & \multicolumn{2}{l|}{} \\
\hline latin & Italien & français & parlers du Nord de l'Italie & toscan $^{13}$ \\
\hline anas & Anade & cane & ànara, ànada & anitra \\
\hline anguis & Bissa & couleuure & bissa & biscia \\
\hline bos & Buo & beuf & buò & bue \\
\hline mendacium & Busia & menterie & busìa & bugia \\
\hline Obliuisci & Dismetigrere & oublier & desmentegàre & dimenticare \\
\hline filius & Fiolo & Filz & fiòlo & figliuolo \\
\hline ebrius & Imbriago & ivrongne & inbriàgo & briaco \\
\hline auis & Losello & oyseau & osèlo & meno \\
\hline farciminator & Lardarolo & saucisier & lardarolo & salsicciaio \\
\hline minus & Mancho & moins & manco & \\
\hline
\end{tabular}

30 Relevons quelques tendances typiques du vénitien : des phénomènes

31 - d'aphérèse de A :

\begin{tabular}{|l|l|l|l|}
\hline \multicolumn{3}{|l|}{ Vocabulaire de trois langues } & \\
\hline latin & italien & Français & Toscan \\
\hline Amicitia & Mistade & Amitye & amistà, amicizia \\
\hline Homicida & Sassino & Meurtrier & Assassino \\
\hline
\end{tabular}

32 - d'apocope du phonème atone ou d'une syllabe en fin de mot :

\begin{tabular}{|l|l|l|l|}
\hline \multicolumn{2}{|l|}{ Vocabulaire de trois langues } & \\
\hline latin & Italien & Français & Toscan \\
\hline \hline Apostoli & Apostol & les apostres & Apostoli \\
\hline \hline Baro & Baron & Baron & Barone \\
\hline \hline Mundine & Merca & Marche (foyre) & Mercato \\
\hline
\end{tabular}


33

- de réduction des géminées :

\begin{tabular}{|l|l|l|l|}
\hline \multicolumn{3}{|l|}{ Vocabulaire de trois langues } & \\
\hline latin & Italien & Français & Toscan \\
\hline \hline Rostrum & Becho & Bec & Becco \\
\hline \hline genu & Genochio & Le genoul & Ginocchio \\
\hline
\end{tabular}

Nombreux sont les vénétismes lexicaux :

\begin{tabular}{|l|l|l|l|}
\hline \multicolumn{2}{|l|}{ Vocabulaire de trois langues } & \\
\hline latin & Italien & Français & Toscan \\
\hline Patruus & Barba & Oncle & zio $^{14}$ \\
\hline Biturum & Bituro & Beurre & burro $^{15}$ \\
\hline
\end{tabular}

Ces modestes remarques peuvent-elles nous aider à mieux dater le Vocabulaire? En effet la variété linguistique que je viens de relever permettrait d'anticiper de quelques années le terminus a quo établi dans Bingen / Van Passen 1991 : le vénitien a disparu assez tôt des Vocabulista ; rappelons que la profonde révision du Vocabulista voulue par Francesco Garrone en 1526 a fait date et que toutes les éditions postérieures en découlent, y compris la première édition française, celle de Lyon 1533. Elles ont donc recours à cette langue commune qu'on est en train de chercher un peu partout en Europe (il suffit de penser à Luther), quête d'où viendra bientôt la standardisation via la fixation. Une des sources de notre Vocabulaire pourrait donc être l'Introductio de Rome 1510 (qui grouille encore de vénétismes, malgré le lieu d'édition : Mazzocchi était originaire de Bergame) ou un de ses descendants le plus proche, les éditions italiennes et allemandes des années 1510-1522.

Il vaut la peine de souligner la grande curiosité des libraires-imprimeurs parisiens pour l'italien, curiosité encore exempte de tout purisme, bien que Pietro Bembo (lui-même vénitien) dans les Prose della volgar lingua (1525) ait déjà sonné le glas pour les langues régionales.

37 Nous en trouvons confirmation - entre autres - dans un recueil de proverbes intitulé Bonne reponse a tous propos, publié en 1547 par Arnoul l'Angelier et par le poète et historien Gilles Corrozet, le grand imprimeur de romans bilingues, qui retraduit personnellement de l'italien le roman de Juan de Flores mentionné plus haut (sous un nouveau titre : Histoire d'Aurelio et Isabelle) et qui remercie, en 1542, Raymond Sac de l'avoir aidé. Or, Raimondo Sacco est originaire de Casei Gerola, village de la province de Pavie $^{16}$.

38 Ce recueil qui a connu un nombre prodigieux d'éditions présente les mêmes traits régionaux de notre Vocabulaire:

Documents pour l'histoire du français langue étrangère ou seconde, 42 | 2009 


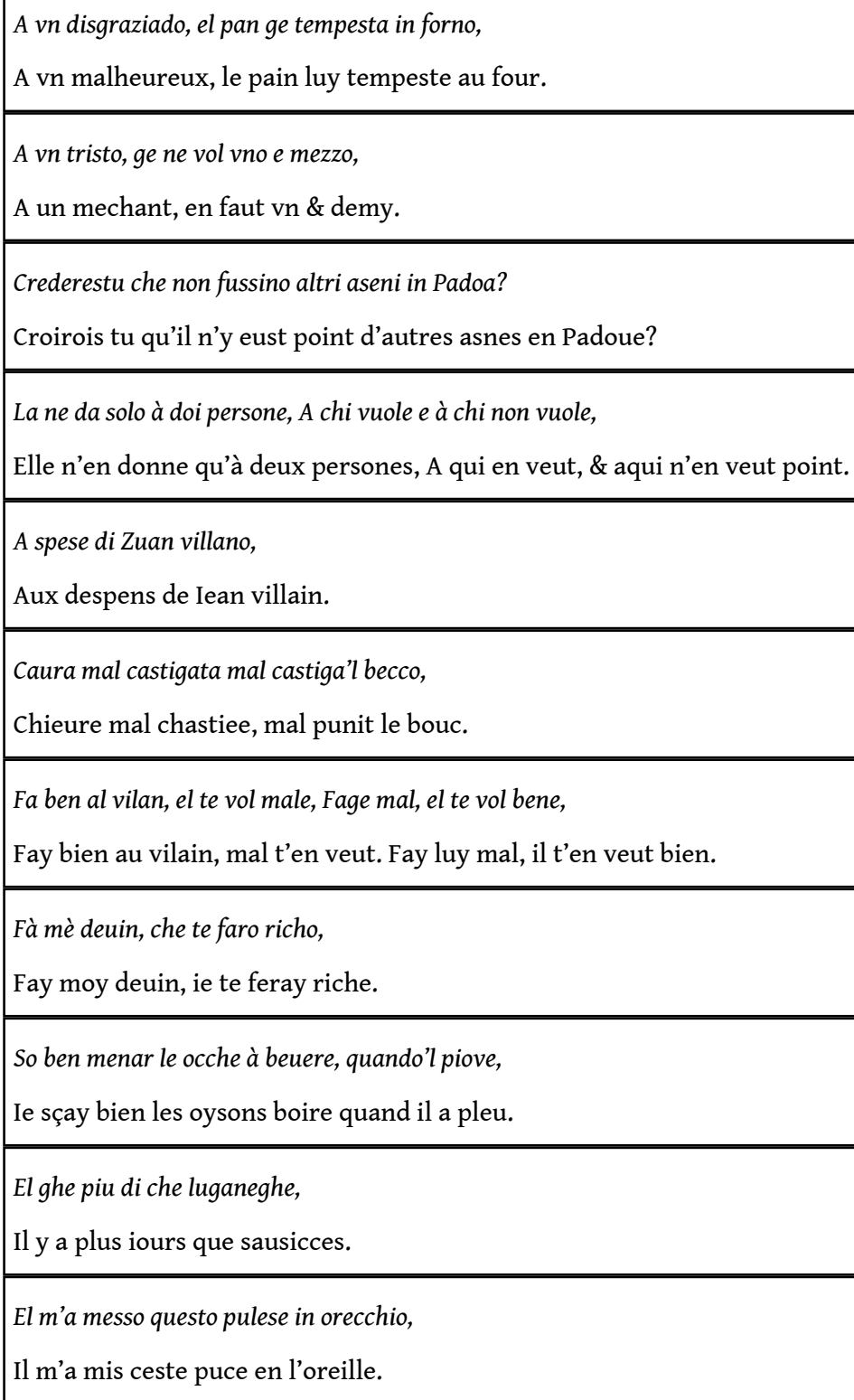

\section{Un outil, son auteur et ses utilisateurs}

La production polyglotte répond toujours à de besoins sociaux. On peut se demander donc si parmi les objectifs du Vocabulaire figurent aussi des finalités éducatives. Le but pédagogique est annoncé dans la page de titre: rappelons que les destinataires mentionnés sont ceux qui désirent apprendre l'italien et le français. Mais le but pédagogique pourrait aussi être inféré de la présentation sur colonnes, selon une pratique qui, à partir du XVI ${ }^{\mathrm{e}}$ siècle, a été appliquée aux manuels du genre Vocabulista ainsi qu'aux textes littéraires ayant ce même but quant à l'apprentissage des langues (pensons, à titre d'exemple, aux éditions polyglottes du Jugement d'amour (voir note 6).

On peut se demander enfin qui est le compilateur de notre Vocabulaire. L'analyse de la nomenclature nous fait pencher pour un Italien, du fait de l'absence de plusieurs équivalents français et latins : si aucun mot traducteur n'est trouvé dans la langue de 
sortie, une périphrase ou une définition sommaire en tient lieu. Voici quelques gloses explicatives ou définitoires:

41 Aierno (arbre duquel ne scay le nom tant francoys que latin)

Bosena Pays ou nom de terre

mantilla Mappe a essuyer les mains

molton certaine beste

Prossia cest nom de pays

sensale nom daulcun mestier

Dans un article intitulé « Des livres pour l'Europe? Réflexions sur quelques ouvrages polyglottes (XVI ${ }^{\mathrm{e}}$ siècle-début XVII ${ }^{\mathrm{e}}$ siècle)", Michel Simonin (1982) avance une réflexion d'un grand intérêt pour la présente enquête : l'Europe des langues existe, tant qu'aucune langue ne s'est encore imposée; au moment où l'une des langues d'Europe parvient à primer sur les autres comme véhicule commun, la production polyglotte se tarit.

Pour ma part, mon incursion dans un outil plurilingue me permet d'ajouter qu'un réductionnisme linguistique interne aux langues va de pair avec ce déclin de la polyglossie. Deux mouvements complémentaires se dessinent : la sélection des langues (langue qui aspire à devenir la langue commune / langues régionales circulant librement avant la vague de la standardisation) et la sélection linguistique (lexique, formes morpho-syntaxiques).

Pour conclure, peu de certitudes, beaucoup de suggestions... des portes qui s'ouvrent sur la galaxie de l'activité linguistique du XVI siècle : voilà enfin une donnée incontestable. Ce dynamisme peut encore nous réserver des surprises.

\section{BIBLIOGRAPHIE}

ACCARISI, Alberto. 1543. Vocabolario, grammatica, et orthographia de la lingua volgare [...]. Cento : In casa dell'Autore.

ALUNNO, Francesco. 1543. Le ricchezze della lingua volgare. Venezia : Aldo Manuzio.

BINGEN, Nicole. 1987. Le maître italien (1510-1660). Bibliographie des ouvrages d'enseignement de la langue italienne destinés au public de langue française [...]. Bruxelles : Émile Van Balberghe.

BINGEN, Nicole, VAN PASSEN Anne-Marie. 1991. «La lexicographie français-italien, italienfrançais ». in F.J. Hausmann, et al. (dir.), Worterbücher, Dictionaries, Dictionnaires [...]. Berlin, NewYork : De Gruyter, 1989-1991, 3007-3013.

BOERIO, Giuseppe. 1993. Dizionario del dialetto veneziano. Firenze : Giunti.

Bonne reponse à tous propos. 1547, Paris : Arnoul l'Angelier / Gilles Corrozet.

CATACH, Nina. 1968. L'orthographe française à l'époque de la Renaissance (Auteurs, Imprimeurs, Ateliers d'imprimerie). Genève : Droz. 
Catalogue de la bibliothèque de feu M. le baron Jérôme Pichon. 1897-1898. Préface de Georges Vicaire,Paris : H. Leclerc et P. Cornuau, 3 vol.

CLAES, Frans. 1977.Bibliographisches Verzeichnis des deutschen Vucabulare und Wörterbücher gedruck bis 1600. New York : Hildeshaim.

COLOMBO TIMELLI, Maria. 1992. «Dictionnaires pour voyageurs, dictionnaires pour marchands, ou la polyglossie au quotidien aux $\mathrm{XVI}^{\mathrm{e}}$ et XVII ${ }^{\mathrm{e}}$ siècles ». Linguisticae Investigationes XVI / 2, 395-420.

-1993. « Il francese del Dictionaire des huict langages (Le Tellier, Parigi, 1546) », in Parcours et rencontres. Mélanges de langue, d'histoire et de littérature offerts à Enea Balmas. Paris : Klincksieck, I, 133-166.

DESTOT, Arlette. 1977. Un libraire parisien au XVI e siècle. Jehan Bonfons, édition et littérature populaire. Université de la Sorbonne : Mémoire de maîtrise.

FINOLI, Anna Maria. 1989. « ... L'intelligence des mots est intelligence de toutes choses... Italiano e francese nei dizionari plurilingui del sec. XVI », in E. Biancardi et al. dir. 1989. Le culture esoteriche nella letteratura francese e nelle letterature francofone. Problemi di lessicologia e lessicografia dal cinquecento al settecento. Fasano : Schena, 345-349.

------1991. « Italien et français dans l'Utilissimo Vocabulista », in Actes du VI Colloque International sur le Moyen Français. Milano : Vita e Pensiero, I, 61-82.

GIUSTINIANI, Vito R. 1987. Adam von Rottweil, Deutsch-Italianischer Sprachführer / Maìstro Adamo de Rodvilla, Introito e porta. Tübingen : Gunter Narr Verlag.

KEMP, William. 1991. « La première édition du Jugement d'amour de Flores (septembre 1529) publiée par Jérôme Denis avec le matériel de Geoffroy Tory ». Bibliothèque d'Humanisme et Renaissance 53 / 3 : 709-726.

LINDEMANN, Margarete. 1994. Die franzosischen Worterbücher von den Anfangen bis 1600 : Entstehung und typologische Beschreibung. Tübingen : M. Niemeyer.

MINERBI, Lucio. 1553. Dittionario di Ambrogio Calepino dalla lingua latina nella volgare brevemente ridotto.Venezia : A San Luca.

MUSSAFIA, Adolfo. 1864. Monumenti antichi di dialetti italiani. Vienna : I. R. Tipografia di Corte e di Stato.

PACCAGNELLA, Ivano. 1984, Il fasto delle lingue : plurilinguismo letterario nel Cinquecento. Roma : Bulzoni.

QUEMADA, Bernard. 1967. Les dictionnaires du français moderne 1539-1863. Étude sur leur histoire leurs types et leurs méthodes. Paris : Didier.

RENOUARD, Philippe. 1964-. Imprimeurs et libraires parisiens du XVI ${ }^{e}$ siècle. Paris : Service des travaux historiques de la Ville de Paris.

ROSSEBASTIANO BART Alda. 1984. Antichi vocabolari plurilingui d'uso popolare : la tradizione del Solenissimo Vochabuolista.Alessandria: Dell'Orso.

SIMONIN, Michel. 1982. «Des livres pour l'Europe ? Réflexions sur quelques ouvrages polyglottes (XVI ${ }^{\mathrm{e}}$ siècle-début XVII ${ }^{\mathrm{e}}$ siècle) », in La conscience européenne. Paris : ENS de Jeunes Filles, 384-394.

VITALE, Maurizio. 1984. La questione della lingua. Palermo : Palumbo. 


\section{NOTES}

1. Vocabulaire de || trois langues/ cestassavoir latine ita/ || lienne \& francoyse de tant de noms quil || cest peu trouuer/ figuré par Alphabet || Tres utile à ceulx qui ont desir scauoir \|de litalien ou bien du françoys. || Et premierement des noms \| qui se commencent par A. \| Du nom italien et non daul \|tre aumoins frequentement. $\|$ On les vend a Paris en la rue neuf $\|$ ve nostre Dame à lenseigne $\mathrm{S}$. Nicolas.

2. Nicole Bingen n'a sans doute pas vu le Vocabulaire puisqu'elle en cite le titre de manière erronée : «Vocabulaire des trois langues », au lieu de « Vocabulaire de trois langues ».

3. L'ouvrage compte 64 feuillets signés A-H. Il enregistre 1746 lemmes. Nombreuses sont les répétitions.

4. Mithridates, de differentiis linguarum tum veterum, tum quae hodie apud diversas nationes in toto orbe terrarum in usu sunt, Conradi Gesneri Tigurini Obseruationes.

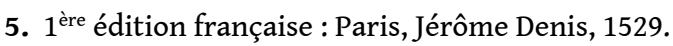

6. À signaler également, dans la seconde moitié $\mathrm{du} X V \mathrm{XI}^{\mathrm{e}}$ siècle, ses 4 éditions bilingues français / espagnol et ses 3 éditions en quatre langues (français / italien / espagnol / anglais) auprès d'éditeurs spécialisés dans les éditions plurilingues, à savoir en colonnes.

7. Introductio quaedam utilissima sive Vocabularius quattuor linguarum Latinae Italicae Gallicae et Alamanicae per mundum versari cupientibus summe utilis. Dans l'Introductio sont ajoutées deux autres langues, le latin et le français, à un vocabulaire bilingue bavarois-vénitien de 1477, l'Introito e porta d'Adam von Rottwil (Adamo de Roduilla). Cf. Giustiniani 1987.

8. Voir Rossebastiano Bart 1984.

9. La plus répandue est celle de l'humaniste Francesco Garrone : Quinque linguarum vtilissimus vocabulista Latine. Tusche. Gallice. Hispane. \& Alemanice. Valde necessarius per mundum versari cupientibus. Nouiter per Franciscum Garonum maxima diligentia in lucem elaboratus... 1526. Toutes les éditions postérieures découlent de cette dernière responsable de métamorphoses importantes tant pour ce qui a trait à l'organisation du lexique que pour la variété linguistique qui sera désormais le toscan.

10. Voir Colombo Timelli 1993. Les 8 éditions parisiennes sont toutes en 8 langues.

11. Les résultats de ce collationnement ainsi que l'analyse linguistique de cette liste lexicale dépasseraient les limites imposées à cette contribution. Ils feront l'objet d'une étude plus vaste.

12. On désigne par «koinè lombardo-veneta (Mussafia 1864) les parlers des Vénéties et de la Lombardie. Ces langues régionales du Nord de l'Italie sont variées et, pour cette époque, il est difficile de les distinguer malgré quelques études de détail sur les nombreux textes littéraires qui nous sont parvenus (textes qui enregistrent les variétés régionales de Bergame, Brescia, Padoue... pour se limiter au Nord) (voir Vitale 1978 : 39-153 et Paccagnella 1984 : passim).

13. Pour les mots toscans, ont été consultés les dictionnaires D'Accarisi (1543), d'Alunno (1543), de Minerbi (1553) et de la Crusca (1612).

14. «Barba. Perzio, detto alla Lombarda » (Vocabolario della Crusca 1612).

15. Biturro est enregistré dans le Vocabolario della Crusca dans une citation tirée de Palladio, originaire de Padoue.

16. Je suis redevable de cette information à Madame Magali Vène, conservateure de la Réserve de la Bibliothèque nationale de France à qui vont mes remerciements. 


\section{RÉSUMÉS}

Le Vocabulaire de trois langues constitue un exemple frappant de contamination entre les deux traditions lexicographiques $\mathrm{du} \mathrm{XVI}^{\mathrm{e}}$ siècle : les dictionnaires polyglottes dits populaires, destinés aux commerçants et aux voyageurs, et les dictionnaires doctes conçus pour les savants. Publié à Paris, difficile à dater, le Vocabulaire est une liste alphabétique sommaire sur trois colonnes ; l'ordre alphabétique est établi sur l'italien qui occupe la colonne centrale. Comme le précise le sous-titre, il est destiné à « ceulx qui ont desir sçavoir de l'italien ou bien du françoys ", deux langues qui entrent en concurrence avec le latin sur la scène européenne. Ce petit dictionnaire trilingue, inconnu jusqu'à présent, est l'occasion pour réfléchir sur les facteurs d'expansion des langues nationales et sur l'intense activité plurilingue qui en constitue le corollaire. Il soulève, d'ailleurs, des questions intra- et extra-textuelles auxquelles on a tenté ici de répondre. Quels sont les rapports de force entre ces trois langues au sein du Vocabulaire? À quel marché éditorial et à quels utilisateurs s'adresse-t-il ? ... La comparaison de sa nomenclature avec celle des autres dictionnaires publiés vraisemblablement dans la même période, ainsi qu'une incursion dans le monde bigarré des imprimeurs nous ont permis de formuler quelques hypothèses.

The Vocabulaire de trois langues is a striking example of contamination between the two lexicographical traditions of the $16^{\text {th }}$ century : the popular multilingual dictionaries for merchants and travellers, and scholarly dictionaries. Published in Paris, but difficult to date, this vocabulary is an alphabetical list on three columns. Alphabetical order is based on the centre column, in Italian. As the subtitle reads, it is intended for « ceulx qui ont desir sçavoir de l'italien ou bien du françoys ", two languages in competition with Latin on the European stage. This little trilingual dictionary, hitherto unknown, is an opportunity to reflect on the expansion of national languages and the intense plurilingual activity which is its corollary. It raises, moreover, intraand extra-textual questions which we tried to answer here. What are the mutual relations among the three languages in the Vocabulaire? What publishing market and users did they address? ... The comparison of its word-list with other dictionaries published probably in the same period, and a foray into the colourful world of printers allow us some interesting assumptions.

\section{INDEX}

Mots-clés : lexicographie plurilingue, XVIe siècle, latin/italien/français, langues nationales, régionalismes, édition française

Keywords : multilingual lexicography, XVIth century, latin/italian/french, national languages, regionalisms, French printers

\section{AUTEUR}

\section{NADIA MINERVA}

Université de Catane 